\title{
Increased Expression of Brain-derived Neurotrophic Factor in Irritable Bowel Syndrome and Its Correlation With Abdominal Pain
}

\author{
(Gut 2012;61:685-694)
}

\author{
Young-Eun Joo \\ Department of Internal Medicine, Chonnam National University Medical School, Gwangju, Korea
}

\section{Summary}

Patients with irritable bowel syndrome (IBS) commonly present with abdominal pain associated with altered bowel habit. Abdominal pain is the symptom most likely to result in medical consultation in IBS patients. ${ }^{1,2}$ Brain-derived neurotrophic factor (BDNF), a member of neurotrophin family, is produced in the central nervous system microglia in response to noxious stimuli and associated with pain modulation and central sensitization. There are large evidences that BDNF may play an important role in visceral pain and hypersensitivity states. ${ }^{3-6}$ Recently, $\mathrm{Yu}$ et $\mathrm{al}^{7}$ investigated the impact of BDNF expression on altered gut sensation in patients with IBS. First, this study was performed in colonic biopsies from 40 patients with IBS and 21 healthy controls. A significant increase in the expression of BDNF and nerve fibers was found in colonic biopsies from patients with IBS, compared with healthy controls. No significant difference was observed in the expression of BDNF and nerve fibers between the IBS subgroups. Transmission electron microscopy showed the ultrastructural damage of nerve axons in the colonic mucosa of patients with IBS. Also, increased expression of BDNF correlated significantly with the severity and frequency of abdominal pain/discomfort in patients with IBS. Next, animal studies using $\mathrm{BDNF}^{+/-}$and $\mathrm{BDNF}^{+/+}$mice were performed to evaluate the role of BDNF in visceral hypersensitivity. The visceral response to colorectal distention (CRD) was evaluated by abdominal withdrawal reflex (AWR) scores. The AWR scores were significantly lowered in $\mathrm{BDNF}^{+/-}$mice at 45 and $60 \mathrm{mmHg}$ CRD pressures applied. The mucosal protein gene product 9.5 was significantly lowered in $\mathrm{BDNF}^{+/-}$mice. Intraperitoneal injection of BDNF induced a significant dose-dependent decrease in threshold pressure and an increased expression of the post-synaptic tyrosine protein kinase $\mathrm{B}(\mathrm{TrkB})$ receptor in the dorsal root ganglia of $\mathrm{BDNF}^{+/+}$mice. Transmission electron microscopy showed the degenerative changes of nerve axons and Schwann cells in $\mathrm{BDNF}^{+/-}$mice. The authors concluded that the increased expression of BDNF with ultrastructural alterations of nerve innervation in the colonic mucosa may contribute to the visceral hyperalgesia in patients with IBS.

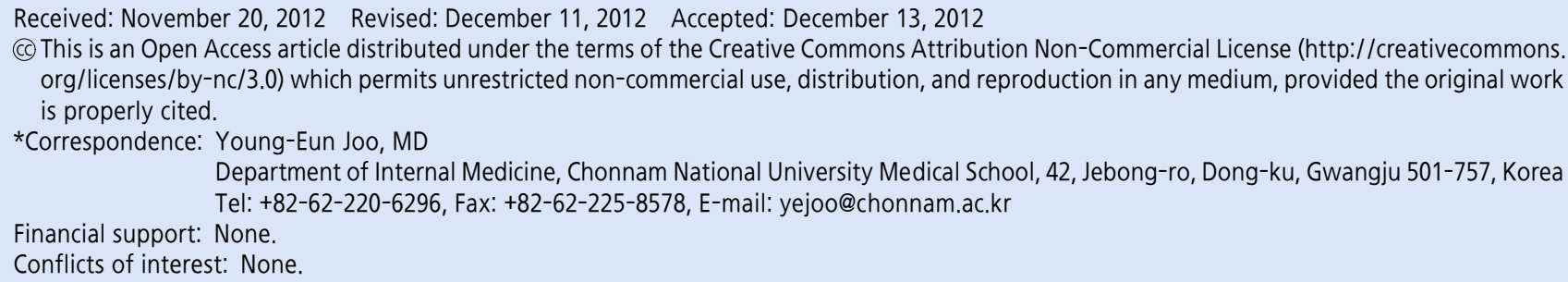




\section{Comment}

IBS is a common gastrointestinal disorder, characterized by recurrent abdominal pain, altered bowel habits and relief of abdominal pain with defecation. ${ }^{1,2}$ The pathophysiology of IBS remains unknown. Many hypotheses have been proposed to explain IBS including alterations in intestinal motility and visceral hypersensitivity, alterations in brain-gut regulatory pathways, postinfectious or postinflammatory changes in digestive neuroimmune function, and alterations in intestinal microflora as possible mechanisms. Among them, mounting evidence suggests that visceral hypersensitivity plays an important role in the pathogenesis of IBS. ${ }^{8-10}$

BDNF is an important neurotrophin, originally described as having developmental and regenerative roles in the nervous system. BDNF has been shown to play a role in animal models of inflammatory hyperalgesia. Also, BDNF leads to local release of sensory neuropeptides which, in turn, activate its effector cell receptors and contributes to the process of chronic pain conditions. ${ }^{3-7}$ Several studies using experimental models of visceral pain $^{4,5}$ and chronic pancreatitis patients with visceral pain ${ }^{6}$ have shown associations between pain and increased uptake of BDNF. Moreover, the inhibition of BDNF reduces visceral sensitivity. However, studies which specifically address any involvement of BDNF in IBS are lacking. Therefore, in this study, the authors investigated the presence of BDNF and nerve fibers in colonic biopsies from patients with IBS and healthy controls, and its relation to the degree of abdominal pain. This study showed increased expression of BDNF and nerve fiber with alterations of nerve innervation in colonic mucosa of patients with IBS and correlation with the abdominal pain score, which may provide a putative basis of IBS symptoms. Previously, BDNF is expressed throughout the gastrointestinal tract in enteric nervous system and mucosa. ${ }^{11,12}$ These data suggest that endogenous BDNF may contribute to visceral pain in patients with IBS.

Although the increased expression of BDNF and nerve fibers were seen throughout the IBS group, there was no significant difference between IBS subgroups when the group was subclassified by Rome II definitions into diarrhea predominant IBS and constipation predominant IBS. The protein level of BDNF was significantly higher in patients with diarrhea predominant IBS than in healthy controls. These results are similar with the previous reports that BDNF stimulates bowel motility and accelerates colonic transit. ${ }^{7,13}$ But the protein level of BDNF in patients with constipation predominant IBS was higher than in healthy controls although not statistically significant. This is strictly a study in a limited number of cases. So, their results are fragmentary, and could not demonstrate the association between BDNF and bowel habit sufficiently. A larger study would probably resolve this issue.

In animal studies using $\mathrm{BDNF}^{+/-}$and $\mathrm{BDNF}^{+/+}$mice, the AWR scores to $\mathrm{CRD}$ and mucosal gene product 9.5 were significantly lowered in $\mathrm{BDNF}^{+/-}$mice. Moreover, intraperitoneal injection of BDNF induced a significant decrease in threshold pressures in $\mathrm{BDNF}^{+/+}$mice. Currently, there are few reports on the mechanism of the visceral hypersensitivity effect of BDNF. Previously, BDNF, synthesized in the dorsal root ganglion, is transported to the central terminals of the primary afferents, is released into the spinal dorsal horn, and binds to $\operatorname{TrkB}$ receptors on second-order sensory neurons in the models of peripheral inflammation and nerve injury. ${ }^{14,15}$ In similar with previous data, the expression of $\mathrm{TrkB}$ receptor in the dorsal root ganglia of $\mathrm{BDNF}^{+/+}$mice was significantly increased by administration of BDNF in this study. These results suggest that BDNF may play a neuromodulatory role in dorsal root ganglia via $\mathrm{TrkB}$ receptor to facilitate pain transmission. Furthermore, transmission electron microscopy showed the ultrastructural damages of nerve axons and Schwann cells in $\mathrm{BDNF}^{+/-}$mice. These results suggest that BDNF deficiency may cause the ultrastructural damages of mucosal nerve innervation and consequently contribute to abnormal visceral sensitivity as the authors described.

In this study, the demonstration of increased expression of BDNF in colonic biopsies from patients with IBS and its correlation with the degree of abdominal pain, may provide a putative basis of visceral pain in patients with IBS. Animal studies using $\mathrm{BDNF}^{+/-}$and $\mathrm{BDNF}^{+/+}$mice showed the involvement of BDNF in visceral hypersensitivity. These findings indicate that increased expression of BDNF may contribute to the visceral pain and hypersensitivity in IBS, and blocking of BDNF may provide a potential therapeutic target for this condition.

\section{References}

1. Longstreth GF. Definition and classification of IBS: current consensus and controversies. Gastroenterol Clin North Am 2005;34: 173-187.

2. Saito YA, Schoenfeld P, Locke GR 3rd. The epidemiology of irritable bowel syndrome in North America: a systematic review. Am J Gastroenterol 2002;97:1910-1915.

3. Obata K, Noguchi K. BDNF in sensory neurons and chronic pain. 
Neurosci Res 2006;55:1-10.

4. Li CQ, Xu JM, Liu D, Zhang JY, Dai RP. Brain derived neurotrophic factor (BDNF) contributes to the pain hypersensitivity following surgical incision in the rats. Mol Pain 2008;4:27.

5. Yang J, Yu Y, Yu H, et al. The role of brain-derived neurotrophic factor in experimental inflammation of mouse gut. Eur J Pain 2010; 14:574-579.

6. Zhu ZW, Friess H, Wang L, Zimmermann A, Büchler MW. Brain derived neurotrophic factor (BDNF) is upregulated and associated with pain in chronic pancreatitis. Dig Dis Sci 2001;46:1633-1639.

7. Yu YB, Zuo XL, Zhao QJ, et al. Brain-derived neutrophic factor contributes to abdominal pain in irritable bowel syndrome. Gut 2012; 61:685-694.

8. Kellow JE, Azpiroz F, Delvaux M, et al. Applied principles of neurogastroenterology: physiology/motility sensation. Gastroenterology 2006;130:1412-1420.

9. Mayer EA, Tillisch K. The brain-gut axis in abdominal pain syndromes. Annu Rev Med 2011;62:381-396.

10. Ohman L, Simrén M. Pathogenesis of IBS: role of inflammation, immunity and neuroimmune interactions. Nat Rev Gastroenterol Hepatol 2010;7:163-173.

11. Maruccio L, Castaldo L, de Girolamo P, Lucini C. Neurotrophin and Trk receptor-like immunoreactivity in the frog gastrointestinal tract. Histol Histopathol 2004;19:349-356.

12. Boesmans W, Gomes P, Janssens J, Tack J, Vanden Berghe P. Brain-derived neurotrophic factor amplifies neurotransmitter responses and promotes synaptic communication in the enteric nervous system. Gut 2008;57:314-322.

13. Coulie B, Szarka LA, Camilleri M, et al. Recombinant human neurotrophic factors accelerate colonic transit and relieve constipation in humans. Gastroenterology 2000;119:41-50.

14. Zhou XF, Rush RA. Endogenous brain-derived neurotrophic factor is anterogradely transported in primary sensory neurons. Neuroscience 1996;74:945-953.

15. Michael GJ, Averill S, Nitkunan A, et al. Nerve growth factor treatment increases brain-derived neurotrophic factor selectively in TrkAexpressing dorsal root ganglion cells and in their central terminations within the spinal cord. J Neurosci 1997;17:8476-8490. 